\title{
Germanica
}

\section{Die zwei Weltkriege in Walter Mehrings Lyrik Vom avantgardistischen Cabaret zur Elegie}

Les deux guerres mondiales dans la poésie de W. Mehring : de l'avant-garde et du cabaret à l'élégie

\section{Monique Boussart}

\section{(2) OpenEdition}

\section{Journals}

Édition électronique

URL : http://journals.openedition.org/germanica/2243

DOI : 10.4000/germanica.2243

ISSN : 2107-0784

Éditeur

Université de Lille

Édition imprimée

Date de publication : 30 juin 2001

Pagination : 119-131

ISBN : 9782913857056

ISSN : 0984-2632

Référence électronique

Monique Boussart, «Die zwei Weltkriege in Walter Mehrings Lyrik Vom avantgardistischen Cabaret zur Elegie », Germanica [Online], 28 | 2001, Online erschienen am: 07 Oktober 2013, abgerufen am 06 Oktober 2020. URL : http://journals.openedition.org/germanica/2243; DOI : https://doi.org/10.4000/ germanica.2243

Ce document a été généré automatiquement le 6 octobre 2020.

(c) Tous droits réservés 


\section{Die zwei Weltkriege in Walter Mehrings Lyrik Vom avantgardistischen Cabaret zur Elegie}

Les deux guerres mondiales dans la poésie de W. Mehring : de l'avant-garde et du cabaret à l'élégie

Monique Boussart

1 Der Name des lange verkannten Satirikers Walter Mehring (1896-1981) ist heute noch vor allem mit der Kabarettszene der zwanziger Jahre verbunden ${ }^{1}$. « Der Bänkelsänger von Berlin », " der schnoddrige Spötter », wie er häufig genannt wurde, bleibt auf die Evokation des hektischen Großstadttempos, auf die Verhöhnung der Weimarer Republik und des Bourgeois festgelegt. Damit werden die düstereren Aspekte und die stilistische Vielfalt seiner Dichtung völlig übersehen.

2 Bei der Untersuchung von Mehrings umfangreichem lyrischem Schaffen fällt auf, dass die Kriegsthematik eigentlich das ganze Werk durchzieht, von den Anfängen im Rahmen des Sturm-Kreises bis zu den traditionelleren, im Exil entstandenen Gedichten. Wie Brecht, mit dem ihn so viel inhaltlich wie formal verbindet, hat der jüdische Schriftsteller zwei Weltkriege durchlebt, den ersten schon als Achtzehnjähriger in Deutschland, den zweiten in der Emigration. Inwieweit der Wandel der politischen Lage und der Lebensverhältnisse seinen Niederschlag findet in der literarischen Vermittlung des Krieges, werden wir im Folgenden aufzuzeigen versuchen.

3 Der Erste Weltkrieg scheint für Mehring wie für so viele Künstler seiner Generation ein Trauma gewesen zu sein, das auch in späteren Jahren tiefe Spuren hinterlassen hat. Kein Wunder : Mehring wurde als Zwanzigjähriger 1916 zum Heeresdienst einberufen ; noch im selben Jahr trat er der Antikriegs-Liga des Harry Grafen Kessler bei, somit bekundete er eine antimilitaristische Position, die er später, um 1930, bestätigte, als er sich der «Gruppe revolutionärer Pazifisten» anschloss, zu der auch Toller und 
Tucholsky gehörten. Wegen «Unzuverlässigkeit» wurde er bald in die «Verdächtigenkompanie » Jüterbog und in die Munitionsfabriken Velten und Spandau eingewiesen.

4 Noch in den dreißiger Jahren werden die politischen Ereignisse immer wieder mit denen von 1914-18 in Zusammenhang gebracht ; die 1932 entstandene Höllische Komödie zum Beispiel gibt ein anschauliches Bild jener Zeit : Sie beschreibt ein Schlachtfeld mit seinen Granatlöchern und den zu Lehmklumpen gewordenen Soldaten, die sich um Gasmasken streiten. Später, im amerikanischen Exil, häufen sich beim Rückblick auf die Kriegsjahre vernichtende Ausdrücke ; Mehring beklagt vor allem die " Mobilmachung » des Geistes, da « alle Lehr-und Lesestätten in Vorschulen der Mordwissenschaft und in Lazarette transformiert, die Professoren zu Instruktionsfeldwebeln avanciert » seien, die die Jugendlichen "als akademisches Kanonenfutter an die Oberste Heeresleitung ablieferten $»^{2}$.

\section{Der Erste Weltkrieg im Zeichen der Avantgarde und des Cabarets}

5 Mehrings frühes Bekenntnis zur Avantgarde, ab 1914-15 zur Wortkunst des Sturm und ab 1918 zu Dada, war ihm auch ein Mittel, sich von der Kriegssituation zu distanzieren. Er schien seine "ganze Hoffnung auf die Niederlage der Tradition gesetzt » zu haben ${ }^{3}$. Jedoch sind seine Sturm-Balladen sowie seine dadaistischen Texte imprägniert von Kriegseindrücken. Dies gilt für manche der in Herwarth Waldens Zeitschrift erschienenen Gedichte, die sich auf den ersten Blick als Evokationen exotischer Sphären zu lesen geben, so zum Beispiel das «Gebet sudanesischer Reiter » (1918), das auf den Schützengrabenkrieg anspielt und deutlich im Gefolge des 1915 gefallenen und vom jungen Mehring verehrten Dichters August Stramm steht. Dies lässt sich sowohl an der assoziativen Schreibtechnik - auch in klanglicher und rhythmischer Hinsicht erkennen als auch am syntaktischen Aufbau des Textes : Er besteht nämlich aus einer schnellen Folge von knappen Verszeilen ohne Interpunktion, oft auf ein einziges Wort, eine Chiffre reduziert. Die sich daraus ergebende staccatohafte Diktion eignet sich besonders gut dazu, die Schockwirkungen und das Chaotische des Kampfes wiederzugeben. Auch in «Brunst der Erde » (1918) klingt Stramms Sprachmanierismus an: Unübliche Wortkombinationen und Wortschöpfungen, die die normale grammatische Kategorie des Wortes verändern, führen zur Verfremdung des herkömmlichen Sprachsystems, analog zu der vom Krieg verursachten Erschütterung und Deshumanisierung. Außerdem greift Mehring $\mathrm{zu}$ einem in solchem Kontext tradierten Verfahren, der Wiederholung von Guttural- und Zischlauten zur Veranschaulichung von Härte und Gefahr :

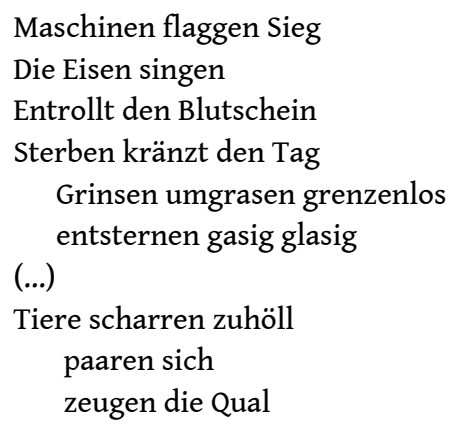


Quälen

Blutverquollen

Seelen lerchen zugott

strahlen

schwindeln

fallen fallen

Schweigen ${ }^{4}$

6 Im Gedicht zum Tode des 1918 gefallenen Sturm-Dichters Wilhelm Runge steigert sich die Verfremdung durch die obsessive Wiederkehr des Lachmotivs, des grellen i-Klangs und des a-Vokals, die den Wahnsinn des Kriegs vergegenwärtigen :

Lachen wirrt in schrecke Augen

Lachen klirrt das Blut zu Eis

(...)

Nahebei stürzt wirft erdenweit krallt ängstetief und zerrt das

Lachen in den Tod

(...) (S. 39f.)

7 Zweifellos lässt sich Mehring hier auf formaler Ebene als Stramm-Jünger, vielleicht sogar als Stramm-Epigone, erkennen, vor allem durch den Willen zur Abstraktion, die Wiederentdeckung des Wortes als Klangkörper und die hermetisch wirkende Unklarheit der grammatischen bzw. syntaktischen Bezüge. Er weicht aber in seiner Kriegsauffassung von seinem Meister ab. Während Stramm, der die Kampfvorgänge als Offizier an der Front unmittelbar erlebte, trotz der Verzweiflung und der Thematisierung von Grauen, Tod und Vernichtung den Krieg doch nicht ohne Ambivalenz darstellt, d.h. ihn gewaltig und großnennt, ist bei Mehring die Ablehnung des Krieges eindeutiger. Die vitalistische Perspektive, die Vorstellung vom Krieg als Ausprägung des Lebens in seinem ständigen Wandel und Werden spielt in seiner Lyrik eine geringere Rolle. Es handelt sich gleichwohl noch um eine enthistorisierte und entpolitisierte Darstellung.

8 Ton und Stil wandeln sich in den Texten, die der Schriftsteller ab 1918 in den DadaBlättern Der blutige Ernst, Die Pleite, Jedermann sein eigener Fussball veröffentlichte ${ }^{5}$. Jetzt sind die Empörung über den Krieg, die Verhöhnung der Reichswehr und des Nationalismus mit bissigen Angriffen gegen die Weimarer Republik, den Untertanengeist, den Heldenkult und die Bolschewistenangst des deutschen Spießers verflochten. Mehrings politische Einstellung hat sich radikalisiert. Nach der Niederschlagung der Novemberrevolution prangert er immer wieder den Verrat der Sozialdemokratie, der Ebert, Scheidemann und Noske an den Proletariern an, die bloß als «Blutlieferanten» (S. 60) behandelt würden. Seine Verse klingen nun viel provokativer ; Klang- und Wortspiele, die Verwendung einer derben Sprache und des Berliner Jargons stehen im Dienst der Verspottung und der linksradikalen Polemik. Der Satiriker experimentiert mit der Collage-Technik, er fügt nicht nur Schlager- und Volksliedfragmente, sondern auch politische Parolen, englische und französische Wendungen in seine Texte ein, in einer Art « Sprachen-Ragtime », wie er sie nennt, eine Technik, der er auch in späteren Gedichten treu bleiben sollte und mit der er seinen eigenen Stil gefunden $\mathrm{zu}$ haben scheint. Besonders charakteristisch ist in dieser Hinsicht « Mordsjubel » (1919), eine Bloßstellung des manipulierbaren, militaristisch und konservativ gesinnten Durchschnittsdeutschen, die in einem sarkastisch hymnischen Ton anhebt und die Propagandasprache als beschönigende Verklärung entlarvt : 
O 1914 !

Du deutscher Frühling

- in Mitte August !

O Jubelrenaissance der Tauentzienstraße -

Feldgrau schwarzweiß !

(...)

Und O Tannenbaum, O Tannenbaum

Die Kriegsweihnacht im Schützengraben

Denn das Volk ist geduldig, bis die Bescherung kommt -

(...)

Bürger, rottet Euch zusammen

Rückt die proletarische Bestie an !

Wir Menschen sind alle gleich

Und Sterben ist süß!

(...) (S. 59)

Meistens bedient sich Mehring der appellativen Struktur und der überspitzten Formulierung des Kabarett-Chansons ${ }^{6}$. Dass der Protest gegen den Krieg noch immer im Mittelpunkt seiner oppositionellen Lieder steht, zeigen folgende Sätze aus der "Conférence provocative», mit der er im November 1919 M. Reinhardts Kabarett «Schall und Rauch » eröffnete :

Antikem Brauchtum gemäß folge dem heroischen Kladderadatsch einer wieder einmal miserablen Geschichtstragödie die schadenfröhliche Satyrfarce; dem Größenwahnwitz der Schmieren-Caesaren der nun spottbillige Galgenhumor; dem Versgerassel der Feste Drauf Gott-strafe-England Barden: der Gassenhauer, die Moritat zum Mordsgaudi der Überlebenden, die gestern noch (Wir alle) dabei gewesen sind, vor Lüttich, Verdun, in den Masurischen Sümpfen, an der Siegfriedlinie $(. . .)^{7}$.

Kennzeichnend ist außerdem der Aufbau des 1920 erschienenen Bandes Das politische Cabaret : Er wird mit dem « Dada-Prolog 1919 » eröffnet, in dem die Folgeerscheinungen der Niederlage angesprochen werden, und mit einer freien Übertragung des anarchistischen Lieds von Charles Davray « Die Fahne» abgeschlossen, eines Plädoyers gegen Nationalismus und Militarismus, das in einen Aufruf zur Desertion mündet.

11 Mehring bedient sich jetzt der typischen Methoden und Stilmittel der Kabarettkunst, insbesondere Mischwortbildungen und Anakoluthe, er bringt disparate Sprachbereiche zusammen und wendet sich der Parodie zu. J. Henningsens Definition des Kabaretts, «Kabarett ist Spiel mit dem erworbenen Wissenszusammenhang des Publikums ${ }^{8}$, könnte der Mehringschen Dichtung jener Jahre nicht besser entsprechen. Man denke an das 1921 veröffentlichte Ketzerbrevier, vor allem an dessen dritten Teil «Die weiße Messe der Häretiker ». Das Eröffnungsstück, eine Litanei, zeichnet sich durch eine besondere Form der Montagetechnik aus, wie aus der letzten Strophe ersichtlich wird:

Kyrie eleison

Dir zum Hohne

Die uns morden

Kreuz auf Orden

Und Kanone

Vom Spitale

Kreuz der Ehre

Von dem Wundmal

Der Misere

(...)

Von dem Blute

Der Soldaten 
Dir zum Hohne morden, Gott

Deinem Sohn und Dir zum Spott

Herr, befreie uns davon

Kyrie eleison! ${ }^{9}$

Griechische oder lateinische Formeln der christlichen Liturgie werden in den deutschen Text eingeschoben, der Schriftsteller setzt liturgische Ausdrucksmittel wie semantische bzw. syntaktische Wiederholungen und Anaphern parodistisch ein, in einem Gebet zur Erlösung von all dem, was zum Krieg gehört oder führt. Damit initiiert Mehring ein literarisches Verfahren, das später in Brechts Hauspostille einen Höhepunkt erreichen sollte. Der ursprüngliche Inhalt wird in sein Gegenteil verkehrt, in einen Angriff auf Religion, Armee und Staat, in einen Aufruf zum Misstrauen gegen diese Instanzen und gegen die «Lammsgeduld (S. 175) überhaupt. Der Satiriker denunziert die von Staat und Kirche scheinheilig tolerierte Unmenschlichkeit des Kriegs und entzaubert somit jene tabuisierten Institutionen.

13 Auf ähnliche Weise finden im «GRADUALE (Dies Irae...)» die Eingangsworte der bekannten Sequenz aus der Totenmesse, "Dies Irae, dies illa», ein klanglich und rhythmisch überzeugendes Äquivalent in «Tag der Rache, Tag der Rache ». Sie leiten hier eine Evokation der Opfer und der Zerstörungen des Krieges ein :

Tag der Rache, Tag der Rache!

Menschen stampfen das : Erwache!

Regimenter Kranke, Schwache!

Krücken klopfen an Skelette

Schutt zerfallener Lazarette

Bäuche federn, weiche, fette

Moder rieseln Mauerritzen

(...)

Bäume wachsen aus Tapeten

Dächer platzen in den Nähten

(...)

Kugeln quer aus den Genicken

Kullern Wagen von den Brücken

Leichen, die vom Zaunpfahl nicken

$(\ldots)^{10}$

14

Sowohl die Motivik als auch die parataktische, elliptische Syntax und lautmalerische Effekte, insbesondere das Vorherrschen der Explosiv-, Dental- und Zischlaute verleihen diesen Zeilen eine Eindringlichkeit, die dem Verismus eines Otto Dix oder der grotesken Unheimlichkeit eines G. Grosz in nichts nachsteht, während der Verweis auf die liturgische Formel die Beschreibung zu einer mahnenden Weltuntergangsvision überhöht, welche hier aber in eine Ankündigung der menschlichen Rache und in eine Mahnung zur Wachsamkeit umschlägt.

Solche bedrückenden Töne wechseln im Ketzerbrevier mit spielerischeren Texten ab. Der Sturz des allzu selbstsicheren Trapezkünstlers im letzten Teil von « Salto Mortale » lässt sich zum Beispiel als Metapher für den Zusammenbruch des Akrobaten Deutschland deuten. Bruchstücke aus dem alten Lied « 0 , du lieber Augustin...» und klischeehafte Wendungen aus der Kriegszeit werden in überstürztem Tempo in Verse 
eingeblendet, die den expressionistischen Reihungsstil, die Strammschen Einwortzeilen mit boshafter Frechheit verbinden :

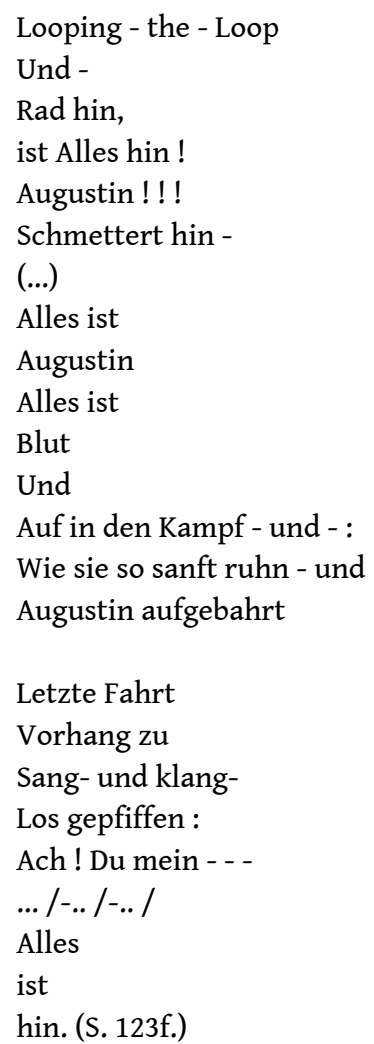

16 Wenn Mehring auch manchmal den Bänkelsang-Ton anschlägt, gibt er doch meistens die übliche narrative Form auf, er reiht eher Bild an Bild, Zitat an Zitat ; der regelmäßig fließende Rhythmus der traditionellen Lyrik wird durch einen "Ragtime ", einen in kurze Explosionen gesprengten Satz ersetzt, der den Leser/Zuhörer irritiert ${ }^{11}$.

\section{Das Fragen nach den Ursachen. Der pamphletische, aufklärerische Duktus}

17 Seit etwa 1923/4 beschränkt sich Mehring nicht mehr auf die Wiedergabe von Eindrücken und auf einen emotionalen Protest, er fragt jetzt nach dem Wie und Warum. Das Kabarettistische und Experimentelle tritt zurück zugunsten einer schlichteren Form, in der jedoch Klang und Rhythmus, scharfe Kontraste sowie umfunktionierte Zitate noch immer einen wesentlichen Platz einnehmen. Hinter den Sarkasmen ist nun der Wille spürbar, die Leser/Zuhörer aufzurütteln, sie vor der Grausamkeit und dem Zynismus der Herrschenden $\mathrm{zu}$ warnen. Einerseits wird die Bestialität des Menschen angeprangert :

Wir zeigen euch die Bestie Mensch gebändigt, Zum erstenmal! Ein Wunder der Dressur! Es riecht nach Wildnis. Hört ihr, wie sie kreischen Ihr « Vive la guerre » und « Immer feste druff»

(Europäische Nächte S. 206)

Andrerseits kommt der zum ersten Mal in Wedding-Montmerte ${ }^{12}$ aufgetauchte Gedanke, dass der Krieg ein Produkt der Entwicklung des Kapitalismus und der den Einzelnen versklavenden Industrie sei, immer schärfer zum Ausdruck. 

«Hopla! Wir leben » hervor, das in das gleich betitelte und von Piscator aufgeführte Drama E. Tollers aufgenommen wurde ; die Großen brauchen "größere Zeiten » und «Säbelrasseln", die Kleinen haben die Folgen zu tragen und die Negierung der revolutionären Ideale, sogar den «Heldentod» hinzunehmen: statt Freiheit und Gleichheit Gitterstäbe und Schützengräben ${ }^{13}$. Bezeichnenderweise wendet sich Mehring jetzt einer Art Rollengedicht zu, das mit plakativen Stereotypen durchsetzt ist. Ein aufschlussreiches Beispiel dafür ist die dritte Variation der « Arie in E-Dur » : « Von den Kriegsnöten ». Auf die Stimmen der Arbeiter, die das Waffenschmieden der Besitzenden als mörderisch entlarven, antworten diese, es sei kein Mord ohne Sinn, er diene Gewinn und Erwerb ${ }^{14}$. Ein ähnliches Gesellschaftsbild tritt uns im "Oratorium von Krieg, Frieden und Inflation » entgegen, das dem ebenfalls von Piscator 1929 aufgeführten Inflationsdrama Der Kaufmann von Berlin vorangestellt und 1931 in das Liederbuch Arche Noah SOS aufgenommen wurde. Der sogenannte Chor in vierhebigen Jamben und Kreuzreimen entpuppt sich, mit seiner zynischen Verfremdung von Parolen der Zeit, als eine Parodie auf Marschlieder, deren charakteristischer Rhythmus durch Anaphern, Klang- und Wortwiederholungen erzeugt wird :

So zogen wir mit der eisernen Ration,

Um im Stahlbad vom Wohlstand zu gesunden -

So zogen wir, von der Mordkommission

Zum Erschießen tauglich befunden -

So zogen wir durch das Brandenburger Tor

(...)

Die letzten Verse jeder Strophe weiten sich zu einer überdimensionalen Vision aus, vor dem grotesk und höhnisch klingenden Refrain :

So stampfte ein Erdteil in gleichem Schritt und Tritt,

Und es zogen Seuchen, Mord und Hunger mit uns mit -

In der schönen, in der neuen, in der schönen, in der neuen

In der schönen, neuen, grauen Felduniform. (S. 329)

Während der Zusammenarbeit mit Piscator wird die Kriegsthematik enger denn je zusammengeflochten mit klassenkämpferischen Motiven; sie ist nun Teil eines ideologischen Pamphlets, von dessen marxistischen Akzenten Mehring sich als anarchistischer Individualist bald wieder distanzieren wird. Dafür gewinnt die Prognose über die Unausweichlichkeit eines neuen Kriegs einen immer bedrohlicheren Ton, der $1929 \mathrm{im}$ aggressiven « Lied der Hakenkreuzler » einen Höhepunkt erreicht :

Und wer nicht gut gewillt

Wird abgekillt und wir marschieren drüber weg

In den frisch-fromm-fröhlichen nächsten Krieg

(...) (S. 335)

\section{Die Rückkehr zu konventionelleren Formen}

Vehementer als früher wird nun der Protest gegen Rassismus und Antisemitismus. Dass der Sozialist Mehring das Leiden der Juden demjenigen der Armen und Enterbten gleichsetzt, hat nichts Verwunderliches an sich. Im Laufe der dreißiger Jahre, in Und Euch zum Trotz (1934) und Staatenlos im Nirgendwo (1934-44), polemisiert er gegen die Ideologie der Rassereinheit, indem er das Nazi-Vokabular in diesem Bereich ironisiert 
und gelegentlich an Heines «Republik der Tiere» anknüpft, zum Beispiel in der sarkastischen Fabel « Vom Maulesel, der sich seiner Abstammung rühmte » ${ }^{15}$.

Der Rückgriff auf Tierfabel und Sage ist für Mehrings damalige Lyrik kennzeichnend. Dessen Funktion scheint weniger die Verschlüsselung als die Intensivierung der Wirkungskraft seiner Angriffe zu sein. So unter anderem in der am Morgen nach dem Reichstagsbrand in der Weltbühne erschienenen "Sage vom großen Krebs » oder in «Der Basilisk - ein Wintermärchen » (1934). Im « heil heil » schreienden Untier ist unschwer der Nationalsozialismus bzw. der Führer $\mathrm{zu}$ erkennen ${ }^{16}$. Die nationalsozialistische Ära wird immer wieder als Rückfall in die Barbarei gebrandmarkt.

In den späten dreißiger Jahren nehmen zwei Themen eine Sonderstellung in Mehrings Schaffen ein: die Appeasement-Politik der Engländer und die Bombardierung Guernicas, die er als Anfang der Greuel betrachtet. "Der Friedensschwatz» löst nochmals seine satirische Verve im Chanson «Ein Friedensnachttraum»(S. 76) aus, während die Niedermetzelung seines Volks durch Franco mit ungewöhnlicher Feierlichkeit und tiefer Empörung in der « Hymne auf den Sieger von Guernica » (S. 78) denunziert wird.

Nicht nur tragische Ereignisse werden festgehalten, auch der Kriegsalltag ist am Anfang des Zweiten Weltkriegs Zielscheibe von Mehrings Ironie, etwa die Beschlagnahmung des Eisens zum Waffenschmieden (S. 88) oder die Huldigungen an den Führer (S. 90). Nicht die Fronterlebnisse, das Heldentum oder das Schicksal der Soldaten, sondern die Angst, die Flucht und das Leiden der Zivilisten, verwüstete Städte und plötzlich abgebrochene Leben stehen im Mittelpunkt metaphernreicher Verse :

Wund im Abendrote

Schwelen die Balken... verglühn

Rostig vom Eisen-Kote

Nordischer Harpyen -

Gräberlose am Platze

Haben ausgewohnt,

Erwürgt vom Krieg beim Schwatze

Unter dem unerlebten Mond...

Wo vor Stunden Etampes noch war...

«Bon soir, Messieurs-dames, bon soir »...

(« La Bonne Dame d'Étampes » S. 136f.)

\section{Zorn und Melancholie des Emigranten}

Im Laufe der Zeit nehmen im Werk des 1933 emigrierten Autors ${ }^{17}$ die Verzweiflung, sogar die Untergangsstimmung und das Bewusstsein seiner totalen «Unzeitgemäßheit » überhand im Vergleich zu dem Protest, insbesondere in den «Briefen aus der Mitternacht» (1938-41), längeren gereimten Gedichten, die den Band No Road back - Kein Weg zurück (1944) eröffnen. Nach Wien geflüchtet, beschwört Mehring Anfang 1938 die Geschwader der deutschen Jagdflugstaffeln über der österreichischen Hauptstadt und den Einmarsch der Deutschen herauf, in der vom « wilden Heer» durchjagten « Rauhnacht $»^{18}$. Wiederholt taucht das Bild vom Reiten « in die Bluthochzeiten» auf; die von Anfang an mit dem Kriegsthema verbundene Höllenmetaphorik und althergebrachte Tiermetaphern gewinnen wieder an 
Bedeutung. Zu Mehrings Bestiarium gehören Termiten, Raben und Heuschrecken, die die Heimsuchung Österreichs und Europas durch die braune Pest veranschaulichen.

Die Irrfahrt der Emigranten, ihre Not in französischen Gefangenenlagern, « eingekäfigt, vogelfrei », da "Schweiß, Lehm und Blut» ihnen "das Kleid» wob ${ }^{19}$, sind nun zum vorherrschenden Gegenstand von Mehrings Texten geworden. In der Silvesternacht 1940/41 kommt er in Marseille, beim Gedenken aller deutschen Dichter, die der NaziHerrschaft zum Opfer fielen, von Mühsam und Ossietzky zu Tucholsky, Toller und Roth, zur elegischen Bilanz : « Der beste Jahrgang deutscher Reben/Ließ vor der Ernte so sein Leben » (S. 129).

Auch wenn die Aggressivität nicht ganz verschwunden ist, so überwiegen doch jetzt Schrecken und Bestürzung; die Trauer hat den Witz verdrängt. Höchst signifikant ist das Gedicht "The March of time »: Der Rückblick auf das Leben des Autors ist eigentlich ein Rückblick auf zwei Weltkriege, zwischen denen eine gescheiterte Revolution, restaurative Tendenzen und «Revanche-Hass " liegen bis zum Auftreten eines « Kavaliers », der alle « mit Barbarei beglückte » (S. 176).

In der Darstellung des Kriegs offenbart sich die ganze Spannweite von Mehrings lyrischer Begabung, die von einer avantgardistischen Diktion zu einer traditionelleren Schreibweise führt. Jedoch sind in der Mannigfaltigkeit einige Konstanten erkennbar : eine außerordentliche verbale Kreativität, die Klang und Rhythmus voll auswertet, das freche Spiel mit der Polysemie und mit intertextuellen Bezügen sowie die Steigerung ins Visionäre. An der Kriegsthematik wird eine Eigenart seines Stils deutlich, nämlich die Verknüpfung des Kabarettistischen und Grotesken mit dem Ernst des Barocks und der Klage um die Vanitas.

Gebrauchslyriker auf seine Weise, ist Mehring dem Phänomen Krieg gegenüber immer gegen jede Faszination und Ambivalenz gefeit geblieben. Keine Sentimentalität, sondern Empörung und Anklage. Der satirische Impuls und die pazifistische Gesinnung siedeln ihn zweifellos in der Nähe Brechts und seines Freundes Tucholsky ${ }^{20}$ an. Vom ersten unterscheidet er sich aber durch seinen Geschichtspessimismus und ein anarchistisch geprägtes Misstrauen gegen das Kollektiv und vom zweiten durch seinen größeren Kampfgeist. Was ihn wiederum mit beiden verbindet, ist die oppositionelle Auffassung der Schriftstellerei als " geistige(r) Entlarvung der Herrschenden »"

\section{NOTES}

1. Für Mehrings Vita und schriftstellerische Entwicklung verweise ich vor allem auf die Monographie von Frank Hellberg: Walter Mehring - Schriftsteller zwischen Kabarett und Avantgarde (Bonn: Bouvier, 1983) und auf die Nachworte von Christoph Buchwald zu den von ihm herausgegebenen Mehring-Bänden im claassen-Verlag. Mein besonderer Dank gilt dem Wissenschaftlichen Archivar des Kabarettarchivs in Mainz, Matthias Thiel, für die freundliche Bereitstellung von Materialien zu Mehring und zum Kabarett.

2. Mehring, W. : Die verlorene Bibliothek. Autobiographie einer Kultur, München : Icking, 1964, S. $180 f$. 3. Ebd. S. 181. 
4. Mehring, W. : Chronik der Lustbarkeiten, Düsseldorf : claassen, 1981, S. 39. Wenn nicht anders vermerkt, sind die folgenden Zitate aus dieser Ausgabe entnommen.

5. Über G. Grosz, mit dem er eng befreundet war, trat Mehring 1918 in Berührung mit den antikapitalistisch und pazifistisch engagierten Berliner Dadaisten um R. Huelsenbeck.

6. Zwischen 1919 und 1921 trat Mehring regelmäßig in Max Reinhardts Kabarett «Schall und Rauch » und in Trude Hesterbergs « Wilder Bühne » auf, deren Berater er damals war.

7. Zitiert nach Karl Riha : Moritat. Song. Bänkelsang. Die moderne Ballade, Göttingen : Sachse u. Pohl, 1965, S. $67 f$.

8. Henningsen, J. : Theorie des Kabaretts, Ratingen : Henn, 1967, S. 24.

9. Mehring, W. : Das Ketzerbrevier, München : K. Wolff, 1921, S. 106f. Eine Neufassung wurde 1974 im Großen Ketzerbrevier publiziert. Sie unterscheidet sich von der ersten durch eine Verallgemeinerung und eine abstraktere Formulierung. Mehring selbst nannte die Texte des Ketzerbreviers « Neobarocklieder », er hielt sie für den wesentlichen Teil seines Schaffens (Brief vom 22. Juli 1961 an Klaus Budzinski im Mainzer Kabarettarchiv).

10. Ebd., S. 109. Bei einem Vergleich mit der späteren Fassung aus 1938 kommt man zu ähnlichen Feststellungen wie für die Litanei. Außerdem zeichnet sich der Text der dreißiger Jahre einerseits durch eine konventionellere Syntax und eine übersichtlichere Gruppierung der Eindrücke aus, wahrscheinlich aus pädagogischen Gründen, andrerseits durch eine Aktualisierung nach der Bombardierung Guernicas, indem die Motorisierung des Kriegs und dessen fantastische Dimensionen hervorgehoben werden.

11. Damit werden auch die Gesetze der thematischen Textprogression und der logischen Konnexionen verletzt, was Benedikt Vogel für ein wichtiges Merkmal der Kabarettliteratur hält : Fiktionskulisse. Poetik und Geschichte des Kabaretts, Paderborn : Schöningh, 1993, S. 130.

12. "Montmerte " kommt aus einem Chanson auf die Butte Montmartre von Aristide Bruant, das der frankophile und damals in Paris weilende Berliner dem Band voranstellte.

13. Mehring, W. : Die Gedichte, Lieder und Chansons des W. Mehring, Berlin : S. Fischer, 1929, S. 14.

14. Ebd., S. 39, 41.

15. Mehring, W. : Staatenlos im Nirgendwo, Düsseldorf : claassen, 1981, S. 49. Wenn nicht anders vermerkt, stammen die folgenden Zitate ebenfalls aus diesem Band.

16. Mehring, W. : Chronik der Lustbarkeiten, A.a.O., S. 417 ; Staatenlos im Nirgendwo, S. 57. Auch Mehrings Prosa ist in dieser Hinsicht aufschlussreich, insbesondere die Umdichtung des Märchens « Rotkäppchen » (1935). Es wird zu einer Allegorie, in der Rotkäppchen das deutsche Volk repräsentiert, das dem Wolf nicht misstraut, von ihm verschlungen wird und sogar den Jäger abweist, der es aus dem Bauch des Wolfs befreien will. In: Mehring, W.: Das Mitternachtstagebuch. Texte des Exils 1933-1939, Mannheim : persona verlag, 1996, S.58ff.

17. Mehring floh zuerst nach Paris, dann nach Wien, 1938 wieder nach Paris und schließlich, 1941 in die USA.

18. Staatenlos im Nirgendwo, A.a.O., S.104-107. In einem 1938 in der Pariser Exilzeitschrift Das neue Tage-Buch erschienenen Prosatext beschreibt der Schriftsteller dieselben Ereignisse : « Als dieser Tag verdämmert, gibt es Wien nicht mehr. Nie zuvor sah ich solch rapiden Physiognomiewandel. Der Hunneneinbruch, der Türkensturm konnten nicht befremdender gewirkt haben (...). Draußen die Finsternis, im Vorbei zerfetzt von den Scheinwerfern, Biwakfeuern anrückender Panzerkolonnen - zur Generalprobe des 'schlagartigen Überfalls'; des 'Ansprungs wie der Blitz aus der Nacht' - auf die Ostmark nun ; auf das Abendland morgen. » (S. 107).

19. Ebd, S. 117,120. Mehring wer selber im Herbst 1939 bis Februar 1940 im Lager Falaise in der Normandie interniert. Im September 1940 wurde er ins Konzentrationslager St. Cyprien, « die Pyrenäenhölle » genannt, gebracht.

20. Tucholsky hat Mehrings Talent sehr früh erkannt. Schon 1920 bezeichnet er ihn in der Weltbühne als den neuen Dichter einer neuen Zeit und rühmt er die rhythmische Kraft seiner Verse. 
21. Mehring, W.: «Die Dreyfusards », in : Die Weltbühne vom 9. September 1930. Zitiert nach Rösler, W. : « Topographie der Hölle », in : Sinn und Form, 1981, Heft 5, S.1119.

\section{RÉSUMÉS}

Die Kriegsthematik durchzieht das ganze lyrische Werk Mehrings; hier entfaltet sich seine stilistische Vielfalt und offenbart sich die Eigenart seines Tons, der Kabarettistisches mit dem Ernst des Barocks verbindet. Den 1918 in der expressionistischen Zeitschrift Der Sturm veröffentlichten Gedichten, die August Stramm verpflichtet sind, folgen in den 20er Jahren provokative, dadaistische Texte, aus denen der Satiriker und Kabarettist spricht. Die Darstellung des Kriegs gewinnt bei ihm jetzt eine politische Dimension; er greift zu einer parodistischen Collage von liturgischen Formeln und politischen Parolen. Bald darauf wird das Thema mit klassenkämpferischen Motiven verflochten, und in der Folgezeit erhebt der Dichter Klage gegen die Möglichkeit eines neuen Kriegs und gegen die Barbarei des Antisemitismus. Mehring bedient sich nun konventionellerer Formen. Melancholie und Trauer haben Aggressivität und Witz verdrängt.

Le thème de la guerre traverse toute l'œuvre de W. Mehring; il fait apparaître la virtuosité stylistique de l'auteur ainsi que la tonalité particulière de sa poésie, qui allie l'humour corrosif du cabaret politique à la gravité du baroque. À ses débuts, placés sous le signe de l'expressionnisme, Mehring exprime ses impressions de guerre dans un style fortement marqué par l'écriture d'August Stramm. Dans les années vingt, Mehring se rapproche du dadaïsme, ses textes, volontiers provocateurs, condamnent la guerre en faisant appel à un montage parodique de formules liturgiques ou de slogans politiques. Par la suite, le thème est de plus en plus lié à celui de la lutte des classes. Peu à peu, la menace d'une nouvelle guerre, la satire de l'antisémitisme nazi, puis l'évocation des villes détruites, du sort des civils et des exilés passent au premier plan, tandis que l'auteur recourt à une forme plus conventionnelle. La mélancolie supplante progressivement l'agressivité.

\section{AUTEUR}

\section{MONIQUE BOUSSART}

Université libre de Bruxelles 